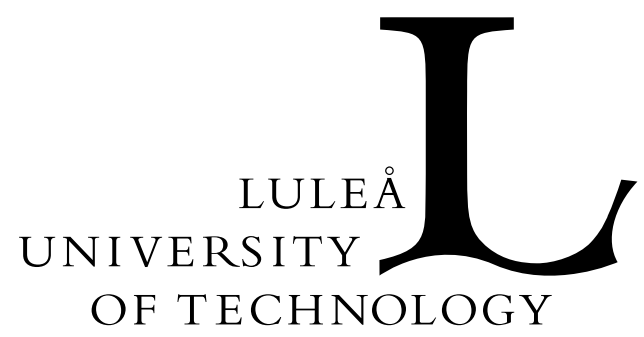

Division of Signal Processing

Visiting address: Universitetsområdet, Porsön, Luleå Postal address: SE-971 87, Luleå, Sweden

Telephone: +46920910 00. Fax: +4692072043

URL: http://www.sm.luth.se/csee/sp/

\title{
Performance Analysis of Coded OFDM on Fading Channels with Non-Ideal Interleaving and Channel Knowledge
}

Magnus Sandell, Sarah Kate Wilson and Per Ola Börjesson,

In Proceedings of Vehicular Technology Conference (VTC 97), Vol. 3, pp. 13801384, Phoenix, USA, May 1997.

(C) 1997 IEEE. Personal use of this material is permitted.

However, permission to reprint/republish this material for advertising or promotional purposes or for creating new collective works for resale or redistribution to servers or lists, or to reuse any copyrighted component of this work in other works must be obtained from the IEEE. 


\title{
Performance analysis of coded OFDM on fading channels with non-ideal interleaving and channel knowledge
}

\author{
Magnus Sandell ${ }^{\dagger}$ \\ Sarah Kate Wilson ${ }^{\ddagger}$ \\ Per Ola Börjesson ${ }^{\dagger}$ \\ $\dagger$ Division of Signal Processing \\ Luleå University of Technology \\ $\ddagger$ School of Electrical and Computer Engineering \\ Purdue University \\ S-971 87 Luleå \\ SWEDEN \\ email: magnus,pob@sm.luth.se \\ Lafayette, IN 47907 \\ U.S.A. \\ email: skwilson@ecn.purdue.edu
}

\begin{abstract}
In this paper we investigate the coded bit-error rate (BER) in an orthogonal frequency-division multiplexing (OFDM) system in a Rayleigh-fading channel. The system uses a pilot-based channel estimator and the effects of non-ideal channel knowledge and non-ideal interleaving are analysed. The coded BER is estimated with an analytical method proposed by Cavers and Ho, which involves truncation of the union bound. For channels with several propagation paths the estimated BER agrees well with simulations. The transfer function bound may however in some cases diverge, thus reducing the accuracy of the estimated BER. We show that this can be the case for channels with few propagation paths.
\end{abstract}

\section{Introduction}

Orthogonal frequency-division multiplexing (OFDM) is an emerging technique for wireless communication. It is used in Europe in digital audio broadcasting (DAB) [1] and is proposed for digital video broadcasting [2]. Its resistance to multipath fading has shown it to be useful in broadcasting applications and it is currently also considered for multiuser systems [3].

For coherent systems, channel estimation is needed. They are usually evaluated by their mean-squared error performance. However, in a communication system the average bit-error rate (BER) is a more relevant measure. The BER can be obtained by simulations but that is time consuming and offers little or no insight to the design problem of a channel estimator. There are analysis tools for coded systems with non-ideal interleaving and channel estimation. The technique derived in $[4,5]$ involves finding the probability of an error event in the decoder and truncating the union bound. However, as pointed out in [6], the union bound may diverge and a good estimate of the BER may not be found by truncating it. We investigate this technique for coded OFDM systems with interleaving across the tones and illustrate the accuracy of this estimation technique for several channels.

The model of the OFDM system, the channel estimator structure and the code is described in Section II. In Section III the error analysis method is described and in Section IV the results from the analytical method are compared with simulations. Finally in Section V the results are discussed.

\section{System description}

\section{II.A OFDM model}

An OFDM system decomposes the available bandwidth into $N$ overlapping narrow frequency bands [7]. The effective symbol length is $T=N T_{s}$, where $T_{s}$ is the sampling period of the system. The channel $g(\tau ; t)$ is assumed to be slowly fading and we consider it to be constant during one OFDM symbol. Furthermore, we assume that the use of a cyclic prefix (CP) [7] of length $T_{c p}$ both preserves the orthogonality of the subchannels and eliminates intersymbol interference (ISI) between consecutive OFDM symbols. In this case we can describe the system as a set of parallel Gaussian channels [7], see Fig. 1. The received signal on

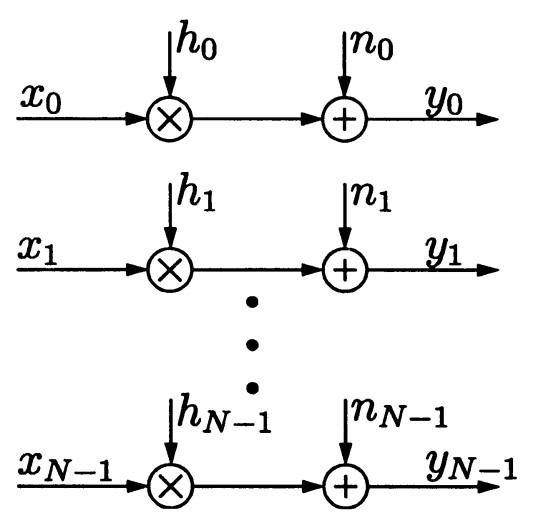

Fig. 1: Model of an OFDM system as a set of parallel channels. 
subchannel $k$ can thus be described as

$$
y_{k}=h_{k} x_{k}+n_{k},
$$

where $x_{k}$ is the transmitted symbol, $n_{k}$ is additive channel noise and

$$
h_{k}=G\left(\frac{k}{N T_{s}} ; t\right), k=0 \ldots N-1,
$$

is the attenuation at subcarrier $k$ and $G(f ; t)$ is the frequency response of the channel $g(\tau ; t)$ during the OFDM symbol at time $t$.

\section{II.B Scenario}

The system we are considering is a wireless multiuser system, e.g. a third generation mobile telephone system. It is operating at the $2.2 \mathrm{GHz}$-band with a bandwidth of 5 $\mathrm{MHz}$ and 1024 subcarriers. This means that the intertone spacing is $4.88 \mathrm{kHz}$ and the symbol duration (excluding the cyclic prefix) is $T=205 \mu \mathrm{s}$. The length of the cyclic prefix is chosen to be $T_{c p}=10 \mu \mathrm{s}$, which makes the effective OFDM symbol length $215 \mu \mathrm{s}$. In this paper we assume perfect synchronization between transmitter and receiver. We will only deal with the downlink although the methods are general and are applicable to the uplink as well. The system is operating in an environment that can be characterized by a Rayleigh-fading channel with a maximum Doppler frequency of $f_{D}=240 \mathrm{~Hz}$ (corresponding to 120 $\mathrm{km} / \mathrm{h}$ ) and a maximum time dispersion of $\tau_{\max }=10 \mu \mathrm{s}$. Normalized to the intertone spacing and sampling interval respectively, this means 5\% maximum Doppler frequency and 50 samples delay spread.

The impulse response of the channel at time $t$ is modelled as

$$
g(\tau ; t)=\sum_{m=1}^{M} \alpha_{m}(t) \delta\left(\tau-\tau_{m}\right),
$$

where the fading amplitudes $\alpha_{m}(t)$ are independent, complex Gaussian random variables and the delays $0<\tau_{m}<$ $\tau_{\max }$ are fixed. This dispersion is shorter than the length of the cyclic prefix and hence ISI is avoided. The amplitudes $\alpha_{m}(t)$ are fading according to the Jakes spectrum [8].

In this paper we will use frequency interleaving on a symbol level, i.e. interleaving is performed after the bits have been assigned to constellation points. The symbols are block interleaved such that two consecutive symbols are separated by 32 subcarriers after interleaving.

\section{II.C Channel estimation}

To estimate the channel we use two-dimensional pilotsymbol assisted modulation as described in [9]. Pilot symbols, known to the receiver, are multiplexed into the transmitted symbol stream. The fading channel is estimated by interpolating between these pilot symbols. The number and positions of the pilots, depicted in Fig. 2, were chosen after some trial-and-error investigation to be a good trade-off between data rate and bit-error probability. To keep the complexity low, separable estimators [9] are used. First the channel attenuations on all subcarriers are estimated in only those OFDM symbols that contain pilot symbols, see Figure 2. Then all channel attenuations are

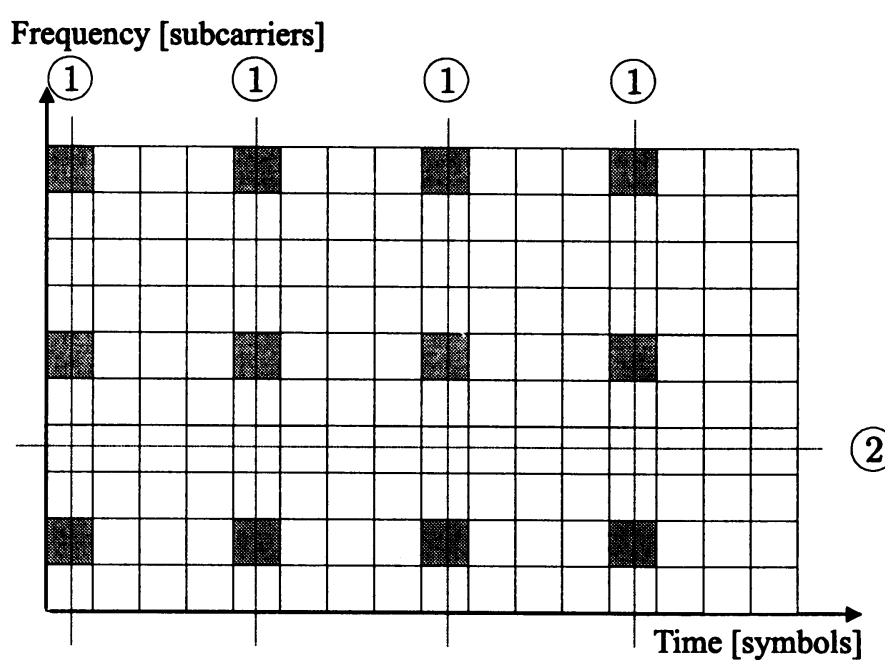

Fig. 2: Pilot symbols, marked in gray, used for channel estimation. The channel attenuations are first estimated in the frequency direction (1) and then in the time direction (2).

estimated using these estimates.

The separate channel estimators are FIR-filters [9] with 5 taps in the frequency direction and 2 taps in the time direction. This estimator has an average complexity 3.25 multiplications per estimated attenuation. The estimators are fixed and designed for nominal values of channel correlation and SNR. Design for the worst case has shown to be robust and beneficial with regard to the maximum error level $[9,10]$. In this paper the estimators are designed for a uniform power-delay profile (for $0<\tau<\tau_{\max }$ ), $S N R=30$ $\mathrm{dB}$ and $5 \%$ maximum Doppler frequency.

\section{II.D Coding and modulation}

For error protection, a convolutional code is used. To make the analysis tractable, a 4-state code with constraint length 3 is used, which has been found to be very effective on fading channels [11]. The in-phase and quadrature signals are encoded separately $[9,11]$ with a rate $1 / 2$ code with generator polynomials:

$$
\begin{aligned}
& g_{0}^{(0)}(D)=1+D+D^{2} \\
& g_{0}^{(1)}(D)=1+D^{2}
\end{aligned}
$$

The encoded outputs are modulated using $4 \mathrm{PAM}$, see Fig. 3 , and concatenated to a $16 \mathrm{QAM}$ symbol. For a fading channel the diversity order of a code is not the free distance but the effective length of the shortest error event [12]. An error event is defined as a path in the trellis that starts at the same node as the correct path, diverges and re-emerges some stages later with the correct path. The effective length of an error event is the number of symbols on 

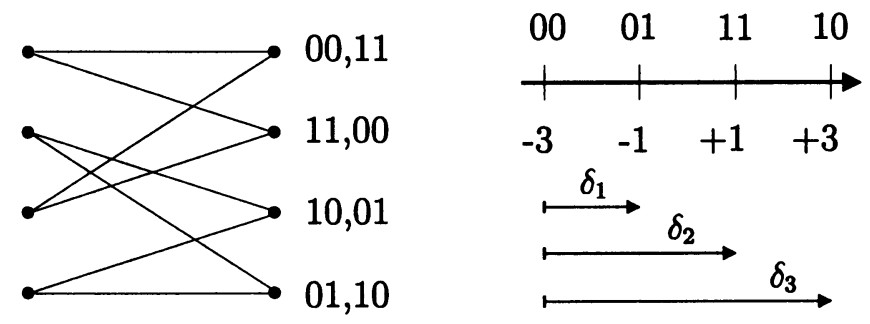

Fig. 3: Trellis of the convolutional code and the 4PAM constellation used in the OFDM system.

an erroneous path that differs from the correct path. The code in (4) with 4PAM modulation has a shortest error event with effective length $L_{\min }=3$ [13].

The receiver first equalizes the received signal $y_{k}$ using the channel estimate $\widehat{h}_{k}$ and then separates the signal into its in-phase and quadrature parts

$$
z_{k}=\frac{y_{k}}{\widehat{h}_{k}}=z_{k}^{(I)}+j z_{k}^{(Q)} .
$$

The decoding is performed separately with the decoding metric

$$
M(\widetilde{\mathbf{x}})=\sum_{k}\left|\widehat{h}_{k}\right|^{2}\left|\widetilde{z}_{k}-\widetilde{x}_{k}\right|^{2},
$$

where $\widetilde{x}_{k}$ is the sequence for which the metric is computed and $\widetilde{z}_{k}$ is either $z_{k}^{(I)}$ or $z_{k}^{(Q)}$. Using the Viterbi algorithm, the sequence $\widehat{x}_{k}$ that minimizes this metric is found. It should be noted that the metric in (5) is not the maximum likelihood metric when multiamplitude modulation is used [4], but we will nevertheless use it for simplicity.

\section{Performance analysis}

To estimate the probability of error for a code, we will use the analysis introduced by Cavers and Ho [4]. Section III.A and III.B describe their technique of calculating the probability of an error event and also how to enumerate them. Section III.C describes the channel correlation for the system in this paper.

\section{III.A Bit-error probability}

Cavers and Ho's analysis is as follows: the average bit-error probability is estimated by truncating the union bound, i.e.

$$
P_{b} \approx \frac{1}{n} \sum_{j} m_{i j} \operatorname{Pr}\left(\mathbf{c}_{i} \rightarrow \mathbf{c}_{j}\right)
$$

where the sum is over a (finite) number of error events and $\operatorname{Pr}\left(\mathbf{c}_{i} \rightarrow \mathbf{c}_{j}\right)$ denotes the probability that the transmitted sequence $\mathbf{c}_{i}$ has larger metric than $\mathbf{c}_{j}$, i.e. an error event will occur. Note that $\mathbf{c}_{i}=\left(\begin{array}{lll}c_{i 1} & \cdots & c_{i L}\end{array}\right)$ contains transmitted symbols $x_{k}$, see Sec. II.A, but in the order given by the interleaving pattern. The number of information bits associated with the error event is denoted by $m_{i j}$ and $n$ is the number of bits per symbol. The error event probability is [4]

$$
\operatorname{Pr}\left(\mathbf{c}_{i} \rightarrow \mathbf{c}_{j}\right)=-\sum_{\text {RHP poles }} \text { Residue }\left[\frac{\Phi_{D}(s)}{s}\right]
$$

where $D$ denotes the difference in metrics between the two sequences $c_{i}$ and $c_{j}$ and $\Phi_{D}(s)$ is the two-sided Laplace transform of its probability density function. The sum is over the poles in the right halfplane $(\operatorname{Re}>0)$ and the poles can be found from eigenvalues of the matrix RM. The matrices $\mathbf{R}$ and $\mathbf{M}$ are defined and discussed in the following two sections.

\section{III.B Error events}

The matrix $\mathbf{M}$ is determined by the code,

$$
\mathbf{M}=\left(\begin{array}{ll}
0 & \mathbf{C}_{j}-\mathbf{C}_{i} \\
\mathbf{C}_{j}^{H}-\mathbf{C}_{i}^{H} & \mathbf{C}_{i}^{H} \mathbf{C}_{i}-\mathbf{C}_{j}^{H} \mathbf{C}_{j}
\end{array}\right),
$$

with $\mathbf{C}_{i}=\operatorname{diag}\left\{c_{i 1}, \ldots, c_{i L}\right\}$ and $\mathbf{C}_{j}=\operatorname{diag}\left\{c_{j 1}, \ldots, c_{j L}\right\}$ and $L$ being the length of the error event. To enumerate the error events we use the technique in [4]. For simplicity the system is analyzed as two separate 4PAM modulated systems and the concatenation to 16QAM symbols is ignored. With the 4PAM constellation, each error can be associated with the distances [14, Chap. 5.3.2]

$$
\begin{array}{ll}
W(00)=0 & W(01)=\delta_{1} \\
W(10)=\frac{1}{2}\left(\delta_{1}+\delta_{3}\right) & W(11)=\delta_{2}
\end{array}
$$

The distance $W(10)$ comes from the fact that when the error 10 occurs, the associated distance will be $\delta_{1}$ when 01 or 11 is transmitted and $\delta_{3}$ when 00 or 10 is transmitted, see Fig. 3. Hence we can denote $W(10)$ by $\frac{1}{2}\left(\delta_{1}+\delta_{3}\right)$ which can be interpreted as $\delta_{1}$ half of the time and $\delta_{3}$ half of the time. Each branch in the error-state diagram, see Fig. 4, is labeled by $W(\cdot) I^{r}$, where $W(\cdot)$ is the Euclidean distance associated with the error and $r$ denotes the number of information bit errors. The error events of length $\leq 6$ are shown in Table 1.

\section{III.C Channel correlation}

The matrix $\mathbf{R}$ denotes the autocovariance matrix of $\left(\begin{array}{ll}\mathbf{y} & \widehat{\mathbf{h}}\end{array}\right)^{T}[15]$, i.e.

$$
\mathbf{R}=E\left\{\left(\begin{array}{c}
\mathbf{y} \\
\widehat{\mathbf{h}}
\end{array}\right)\left(\begin{array}{c}
\mathbf{y} \\
\widehat{\mathbf{h}}
\end{array}\right)^{H}\right\}=\left(\begin{array}{ll}
\mathbf{R}_{y y} & \mathbf{R}_{\widehat{y}} \\
\mathbf{R}_{\widehat{h} y} & \mathbf{R}_{\widehat{h} \widehat{h}}
\end{array}\right),
$$

where $\mathbf{y}=\left(\begin{array}{lll}y_{1} & \cdots & y_{L}\end{array}\right)$ are the received samples and $\widehat{\mathbf{h}}=\left(\begin{array}{lll}\widehat{h}_{1} & \cdots & \widehat{h}_{L}\end{array}\right)$ are the estimates of the channel. The correlations in (7) for the system in this paper can be calculated in the following manner. For the channel model in (3), the correlation function is

$$
\begin{aligned}
R_{G}(\Delta f ; \Delta t) & =E\left\{G(f ; t) G^{*}(f-\Delta f ; t-\Delta t)\right\} \\
& =J_{0}\left(2 \pi f_{D} \Delta t\right) \sum_{m=1}^{M} E\left\{\left|\alpha_{m}\right|^{2}\right\} e^{-2 \pi \tau_{m} \Delta f}
\end{aligned}
$$




\begin{tabular}{|l|l|l|l|l|l|}
\hline $\begin{array}{l}\text { Error } \\
\text { event \# }\end{array}$ & $L$ & $L_{\text {eff }}$ & $\begin{array}{l}\text { Error } \\
\text { event }\end{array}$ & $\begin{array}{l}\text { Bit errors } \\
m_{i j}\end{array}$ & $\begin{array}{l}\text { Euclidean } \\
\text { distances }\end{array}$ \\
\hline \hline 1 & 3 & 3 & $s_{0} \rightarrow s_{1} \rightarrow s_{2} \rightarrow s_{0}$ & 1 & $\delta_{2}, \frac{1}{2}\left(\delta_{1}+\delta_{3}\right), \delta_{2}$ \\
\hline 2 & 4 & 4 & $s_{0} \rightarrow s_{1} \rightarrow s_{3} \rightarrow s_{2} \rightarrow s_{0}$ & 2 & $\delta_{2}, \delta_{1}, \delta_{1}, \delta_{2}$ \\
\hline 3 & 5 & 4 & $s_{0} \rightarrow s_{1} \rightarrow s_{2} \rightarrow s_{1} \rightarrow s_{2} \rightarrow s_{0}$ & 2 & $\delta_{2}, \frac{1}{2}\left(\delta_{1}+\delta_{3}\right), 0, \frac{1}{2}\left(\delta_{1}+\delta_{3}\right), \delta_{2}$ \\
\hline 4 & 5 & 5 & $s_{0} \rightarrow s_{1} \rightarrow s_{3} \rightarrow s_{3} \rightarrow s_{2} \rightarrow s_{0}$ & 3 & $\delta_{2}, \delta_{1}, \frac{1}{2}\left(\delta_{1}+\delta_{3}\right), \delta_{1}, \delta_{2}$ \\
\hline 5 & 6 & 5 & $s_{0} \rightarrow s_{1} \rightarrow s_{3} \rightarrow s_{2} \rightarrow s_{1} \rightarrow s_{2} \rightarrow s_{0}$ & 3 & $\delta_{2}, \delta_{1}, \frac{1}{2}\left(\delta_{1}+\delta_{3}\right), \frac{1}{2}\left(\delta_{1}+\delta_{3}\right), \delta_{1}, \delta_{2}$ \\
\hline 6 & 6 & 5 & $s_{0} \rightarrow s_{1} \rightarrow s_{2} \rightarrow s_{1} \rightarrow s_{3} \rightarrow s_{2} \rightarrow s_{0}$ & 3 & $\delta_{2}, \delta_{1}, \delta_{1}, 0, \frac{1}{2}\left(\delta_{1}+\delta_{3}\right), \delta_{2}$ \\
\hline 7 & 6 & 6 & $s_{0} \rightarrow s_{1} \rightarrow s_{3} \rightarrow s_{3} \rightarrow s_{3} \rightarrow s_{2} \rightarrow s_{0}$ & 4 & $\delta_{2}, \frac{1}{2}\left(\delta_{1}+\delta_{3}\right), 0, \delta_{1}, \delta_{1}, \delta_{2}$ \\
\hline
\end{tabular}

Table 1: Description of the dominating error events. The length of the error event is $L$, the effective length, $L_{e f f}$, is the number of symbols that differs from the transmitted codeword, $m_{i j}$ is the average number of bit errors associated with the error event, and $\delta_{i}$ are the Euclidean distances of the codeword symbols, see Fig. 3.

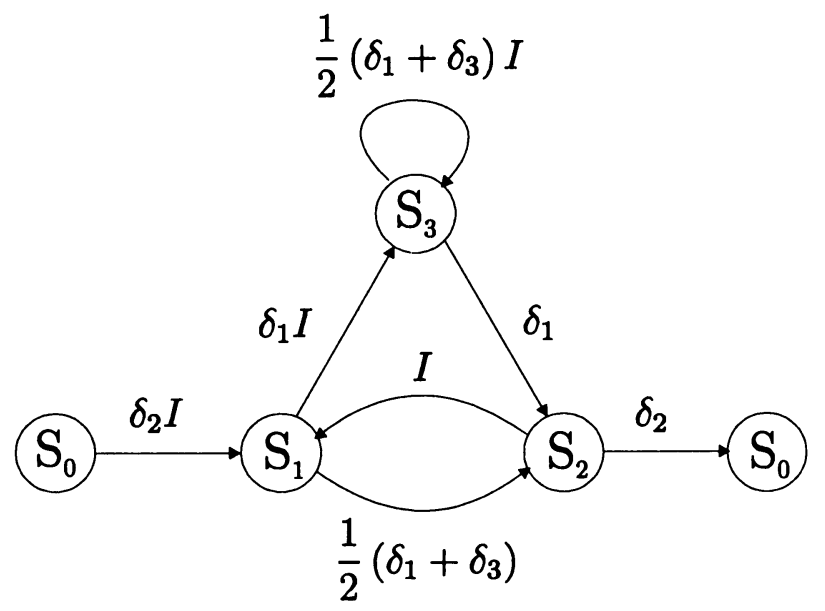

Fig. 4: Error-state diagram for the convolutional code $\left(1+D+D^{2}, 1+D^{2}\right)$-code with 4 PAM modulation.

where $J_{0}(\cdot)$ is the zeroth order Bessel function of the first kind. Each estimate of the channel is a linear combination of the observed channel at the pilot positions. Thus the channel estimate may be written as $\widehat{h}=\mathbf{w}^{H} \mathbf{p}$, where $\mathbf{w}$ is the estimator and $\mathbf{p}$ the observed channel. Using this vector notation it is straightforward to obtain the desired correlations. As an example, take the crosscorrelation

$$
E\left\{y_{k} \widehat{h}_{l}^{*}\right\}=E\left\{\left(h_{k} c_{i k}+n_{k}\right) \mathbf{p}_{l}^{H} \mathbf{w}\right\}=E\left\{h_{k} \mathbf{p}_{l}^{H}\right\} c_{i k} \mathbf{w}
$$

where $\mathbf{p}_{l}$ are the observed channel attenuations used to estimate $h_{l}$. The channel correlations $E\left\{h_{k} \mathbf{p}_{l}^{H}\right\}$ are determined by first finding their relative positions to each other, e.g. $m$ subcarriers and $n$ OFDM symbols, and then using the channel correlation (8)

$$
R_{G}\left(m \cdot \frac{1}{T}, n \cdot\left(T+T_{c p}\right) f_{D}\right)
$$

where $1 / T$ is the intertone spacing and $T+T_{c p}$ is the OFDM symbol length (including the cyclic prefix). Remaining correlations in (8) are found in a similar way. Note that the index $k$ is after interleaving, so the channel attenuations $h_{k}$ and $h_{k-1}$ are separated by 32 subcarriers (see
Sec. II.B). More details on the correlations are available in [13].

\section{Simulation}

We have simulated the described system with the channel model in (3) and the parameters as given in Table 2, where the delays are given in samples.

\begin{tabular}{|l|l|l|}
\hline \# of paths & Delays, $\tau_{m}$ & Powers, $E\left\{\left|\alpha_{m}(t)\right|^{2}\right\}$ \\
\hline \hline 1 & 0 & 1 \\
2 & 0,50 & 1,1 \\
3 & $0,10,50$ & $1,1,1$ \\
5 & $0,10,20,30,50$ & $1,1,1,1,1$ \\
\hline
\end{tabular}

Table 2: Channel parameters

Error events of length $\leq L_{\max }=6$, see Table 1 , were considered. The signal-to-noise ratio is defined as

$$
\mathrm{SNR}=\frac{E\left\{\left|h_{k}\right|^{2}\right\} E\left\{\left|x_{k}\right|^{2}\right\}}{E\left\{\left|n_{k}\right|^{2}\right\} \cdot b}
$$

where $b$ denotes the number of information bits per symbol. The estimated and simulated BER for the 1- and 2-path channel are shown in Fig. 5. For these channels, the BER is overestimated. With few propagation paths, the channel correlation after interleaving is still high. As pointed out in [6], the union bound of the BER may diverge and a good estimate cannot be obtained by truncating it. Note that the diversity of the coded system in a 1 and 2-path channel is less than 3 , the diversity of the code.

For the 3- and 5-path channels, the estimated BER agrees better with the simulations, see Fig. 6 . Here the interleaved channel attenuations are more decorrelated. In this case the truncated union bound provides a better estimate of the BER.

\section{Conclusions}

In this paper we have applied an analysis method [4, 5] to a coded OFDM system. This method is most useful in 


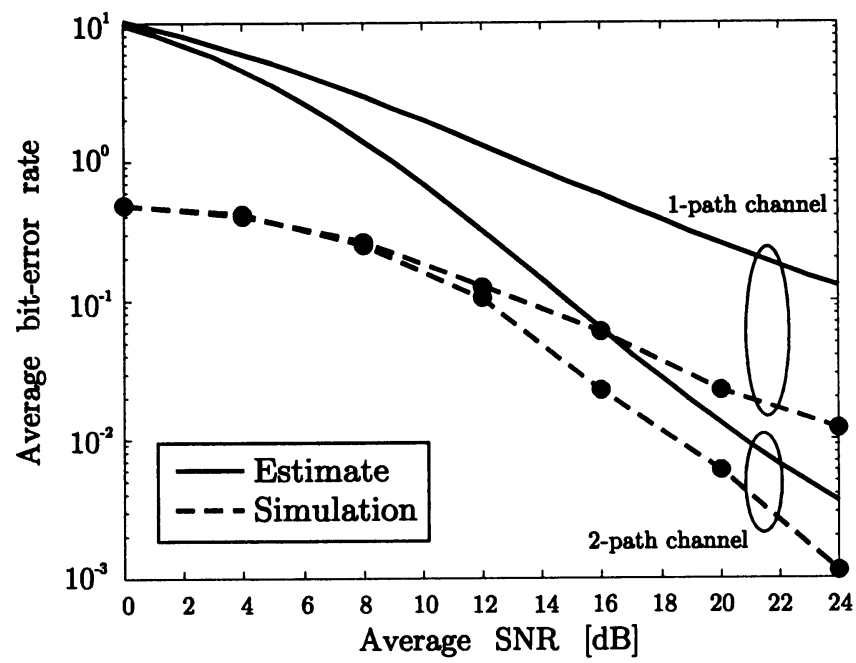

Fig. 5: BER for the 1- and 2-path channels.

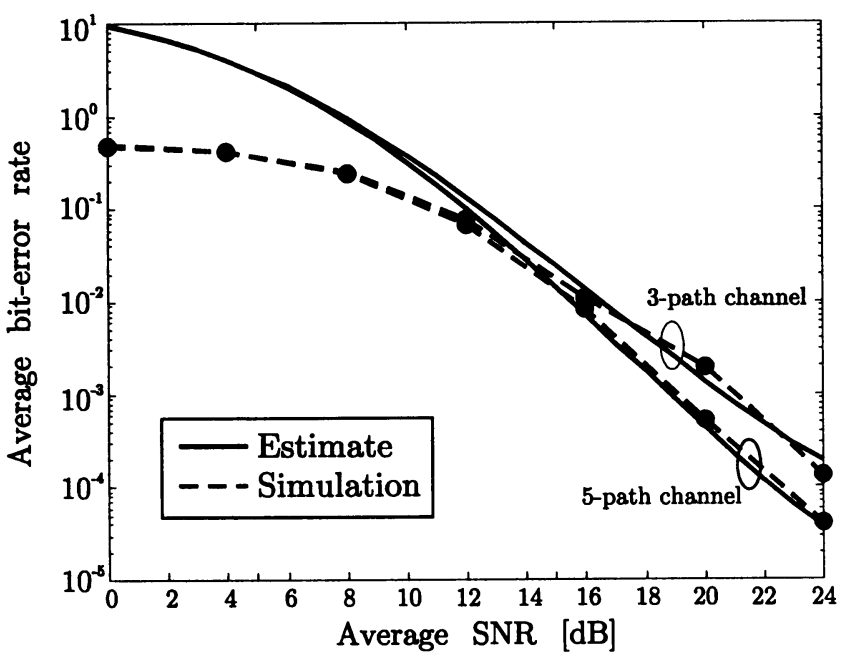

Fig. 6: BER for the 3- and 5-path channels.

the design of a system, with respect to channel estimator complexity, interleaving schemes etc., since it does not require any time-consuming simulations. Furthermore, it is general and allows the analysis to consider systems with non-ideal channel estimation and interleaving. The theoretical results agree well with simulations, except for the channels with few propagations paths. The union bound may in some cases diverge [6] and thus truncating the union bound as in (6) may not give an accurate estimate of the BER. When the channel has several propagation paths, it is possible to decorrelate it with interleaving and the estimated BER agrees better with simulations. In this case the investigated method shows a high potential for use in the design phase of coded OFDM systems, since it is fast and simple. In this paper we have only considered the effect of channel estimation, but other parameters can also be investigated, such as pilot density, choice of code, etc.

\section{References}

[1] Radio broadcasting systems; Digital Audio Broadcasting (DAB) to mobile, portable and fixed receivers. ETS 300 401, ETSI - European Telecommunications Standards Institute, Valbonne, France, February 1995.

[2] Digital broadcasting systems for television, sound and data services. European Telecommunications Standard, prETS 300744 (Draft, version 0.0.3), April 1996.

[3] H. Rohling and R. Grüheid. Multicarrier transmission technique in mobile communication systems. In Proc. RACE Mobile Commun. Summit, pages 270-276, Cascais, November 1995.

[4] James Cavers and Paul Ho. Analysis of the error performance of trellis-coded modulation in Rayleigh-fading channels. IEEE Trans. Commun., 40(1):74-83, January 1992.

[5] Paul Ho and Dominic Fung. Error performance of interleaved trellis-coded PSK modulations in correlated Rayleigh-fading channels. IEEE Trans. Commun., 40(12):1800-1809, December 1992.

[6] Christian Schlegel. Error probability calculation for multibeam Rayleigh channels. IEEE Trans. Commun., 44(3):290-293, March 1996.

[7] John A. C. Bingham. Multicarrier modulation for data transmission: An idea whose time has come. IEEE Commun. Mag., 28(5):5-14, May 1990.

[8] J.G. Proakis. Digital communications. Prentice-Hall, 3rd edition, 1995.

[9] Peter Höher. TCM on frequency-selective land-mobile fading channels. In Proc. Tirrenia Int. Workshop Digital Commun., Tirrenia, Italy, September 1991.

[10] James K. Cavers. An analysis of pilot-symbol assisted modulation for Rayleigh-fading channels. IEEE Trans. Vehic. Technol., 40(4):686-693, November 1991.

[11] Paul Ho, James Cavers, and Jean Varaldi. The effects of constellation density on trellis-coded modulation in fading channels. IEEE Trans. Vehic. Technol., 42(3):318-325, August 1993.

[12] Ezio Biglieri, Dariush Divsalar, Peter J. McLane, and Marvin K. Simon. Introduction to trellis-coded modulation with applications. Macmillan, New York, 1991.

[13] Magnus Sandell, Sarah Kate Wilson, and Per Ola Börjesson. Performance analysis of coded OFDM on fading channels with non-ideal interleaving and channel knowledge. Research Report TULEA 1996:20, Div. of Signal Processing, Luleå University of Technology, September 1996.

[14] S. Hamidreza Jamali and Tho Le-Ngoc. Coded-modulation techniques for fading channels. Kluwer Academic Publishers, 1994.

[15] Robert van Nobelen and Desmond P. Taylor. Analysis of the pairwise error probability of noninterleaved codes on the Rayleigh-fading channel. IEEE Trans. Commun., 44(4):456-463, April 1996. 